\title{
A Sensitive Signal-On Supersandwich DNA Biosensor based on the Enhancement of Poly(aniline-luminol) Nanowires Electrochemiluminescence by Ferrocene
}

Zhifang DING, Yue LI, Ying BAO, Kexin HAN, Iparguli TUREPU and Guixin $\mathrm{LI}^{\dagger}$

Xinjiang Key Laboratory of Energy Storage and Photoelectrocatalytic Materials, School of Chemistry and Chemical Engineering, Xinjiang Normal University, Urumqi, Xinjiang 830054, P.R. China

\footnotetext{
${ }_{\dagger}^{\dagger}$ To whom correspondence should be addressed.

E-mail: 1iguixin2@,163.com
} 


\begin{abstract}
A signal-on supersandwich type of electrochemiluminescence (ECL) DNA biosensor was developed based on the poly(aniline-luminol) nanowires (PALNWs) modified electrode and enhancement of Ferrocene $(\mathrm{Fc})$ on ECL of luminol. Aminated capture DNA was covalently linked to the PALNWs on electrode surface by the crosslinking of glutaraldehyde. In presence of target DNA, its 3' terminus hybridizes with capture probe and the $5^{\prime}$ terminus hybridizes with Ferrocene labeled DNA (Fc-DNA) to form a long DNA concatamer supersandwich structure. The ECL intensity of the prepared biosensor was improved distinctly with increasing concentration of target DNA due to the enhancement of Ferrocene on luminol ECL. The difference of the ECL intensity in the absence and presence of target DNA was used to monitor the hybridization event. The difference of ECL linearly increased with the logarithm of target DNA concentration in the range from $1.0 \times 10^{-16} \mathrm{~mol} \mathrm{~L}^{-1} \sim 1.0 \times 10^{-8} \mathrm{~mol} \mathrm{~L}^{-1}$ with a detection limit of $5.8 \times 10^{-17}$ mol L ${ }^{-1}$. The sensor had high sensitivity and wide linear relationship for the detection of target DNA.
\end{abstract}

Keywords: Electrochemiluminescence, poly(aniline-luminol) nanowires, Ferrocene, Supersandwich, DNA biosensor 


\section{Introduction}

DNA can be used as a biological vector to store and transmit genetic information, and sensitive detection of DNA is quite important in the fields of gene expression analysis, clinical diagnosis, drug research, forensic analysis, and environmental investigation. ${ }^{1,2}$ DNA detection systems is mainly based on the hybridization between a target DNA and its complementary probe. DNA biosensors (also called genosensors) exploits the preferential binding of complementary single-stranded nucleic acid sequences. As is well known, DNA biosensor has good sensitivity and selectivity, thus, various DNA biosensors have been developed for the detection of sequence-specific DNA, including biosensors based on colorimetry, ${ }^{3,4}$ fluorescence, ${ }^{5-7} \mathrm{ECL}^{8,}{ }^{8}$ photoelectrochemistry, ${ }^{10}$ dynamic light scattering technology, ${ }^{11}$ and electrochemistry. ${ }^{12-14}$ Among these methods, ECL has the advantages of high sensitivity, low background, good selectivity, wide linear range, low equipment cost, and has become an attractive and powerful detection tool in the fields of biosensor and bioassay. ${ }^{15}$ For example, Chen et al. established a Signal-off/on ECL deoxyribosensors for assay of early lung cancer biomarker (NAP2) based on target-caused DNA charge transfer. ${ }^{16}$ Chen et al. developed a dual-signalamplified cloth-based ECL biosensor for p53 gene detection, where luminol functionalized gold nanoparticles are used for probe labels and signal amplification. ${ }^{17}$

Luminol is a famous organic luminophore because of its high ECL quantum yield, low excitation potential, low cost, non-toxicity and good water solubility. ${ }^{18,} 19$ Consequently, it has been widely used in diagnostic, clinical, pharmaceutical, environmental, food analysis and other fields. ${ }^{20}$ In order to develop easy-to-use reagentless systems that avoid conventional luminophore addition, the immobilization of luminol on a transducer surface appears attractive, and solid-state luminol ECL sensors based on the immobilization of luminol on electrode have been studied and applied in wide fields. ${ }^{21}$ As an aniline derivative, luminol can be electropolymerized on different electrode surface, which is favorable to the preparation of luminol-based sensor. $^{22}$ Furthermore, luminol-based nanocomposites combined with some polymers have been prepared and used to develop ECL sensors. ${ }^{23-26}$ For example, polyaniline, as one of the most studied conductive polymers that has played an important role in sensor applications, ${ }^{27}$ has been used to copolymerized with luminol to form composite nanowires. ${ }^{25}$ Wang et al. have designed an ultrasensitive ECL biosensor using poly-Llysine as a co-reactant of luminol and poly(luminol/aniline) nanorods loaded reduced graphene oxide as nanoprobe for the detection of glutathione with high sensitivity. ${ }^{25}$ Wei et al. have developed an ultrasensitive electrochemiluminescence immunosensor for alpha-fetoprotein (AFP) detection based on a poly(aniline-luminol) nanowires/graphene oxide nanocomposite. ${ }^{26}$

In this study, PALNWs with stable ECL behavior and excellent film-forming property were prepared with a simple chemical oxidation synthesis. And then, a sensitive supersandwich ECL DNA biosensor was constructed based on the prepared PALNWs. Firstly, PALNWs with numerous amino groups not only provided stable ECL signals, but also served as a matrix to combine amination capture probe (cDNA). Target 
DNA (tDNA) hybridized to two different regions of cDNA and Fc-DNA, forming the sandwich type construction. The Ferrocene on the Fc-DNA can effectively catalyze the ECL of luminol in the PALNWs. When the concentration of tDNA increased, continuous hybridization reaction happened between tDNA and Fc-DNA. Thus, ECL intensity of PALNWs increased with the increasing of concentration of tDNA. The experiment results showed that the ECL intensity of luminol was linear with the logarithm of tDNA concentration.

\section{Experimental}

\section{Reagents}

Luminol was produced from Sigma-Aldrich (St. Louis, Missouri, USA). Aniline sulfate $\left(\mathrm{C}_{12} \mathrm{H}_{14} \mathrm{~N}_{2} \cdot \mathrm{H}_{2} \mathrm{SO}_{4}\right)$ was purchased from Aladdin Biochemical Technology Co., Ltd., Shanghai, China. Ammonium persulfate $\left(\left(\mathrm{NH}_{4}\right)_{2} \mathrm{~S}_{2} \mathrm{O}_{8}\right)$ was obtained from Tianjin Shenggao Chemical Reagent Co., Ltd., Tianjin, China. 50 \% glutaraldehyde (GA) was provided by Chengdu Kelong Chemical Reagent Factory. All buffers and aqueous solution were prepared with ultrapure water $(18.2 \mathrm{M} \Omega \mathrm{cm}$, Millipore). All oligonucleotide fragments were synthesized by Shanghai Sangon Inc., Shanghai, China. The complementary sequence is designed as one fragment of Escherichia coli (E. coli) DNA sequence. Their base sequences were as follows:

Capture probe (cDNA): 5'-NH $-\left(\mathrm{CH}_{2}\right)_{6}$-CTT CCT CCC CGC TGA TAT TAA CTT TAC TCC-3'; Complementary sequence (tDNA): 5'-TCA GCG GGG AGG AAG GGA GTA AAG TTA ATA-3'; Fc labeled sequence (Fc-DNA): 5'-CTT CCT CCC CGC TGA TAT TAA CTT TAC TCC-Ferrocene-3'; Single base mismatch sequence $\left(\mathrm{S}_{1}\right)$ : 5'-TCA GCG GAG AGG AAG GGA GTA AAG TTA ATA-3'; Non-complementary sequence $\left(\mathrm{S}_{2}\right)$ : 5'-ATG CTA TAT GAC TCA ATC CAG GGT CGC TGC-3'; The all oligonucleotide reserve solutions were prepared with $0.01 \mathrm{~mol} \mathrm{~L}^{-1}$ phosphate buffer solution (PBS, $\mathrm{pH}=7.40$ ) and stored in the refrigerator at $4{ }^{\circ} \mathrm{C}$.

\section{Apparatus}

The morphology of PALNWs were characterized by transmission electron microscope (TEM, H-600, Hitachi, Japan). Ultraviolet-visible absorption spectrometer (TU-1901, Puxi Analytical Instruments Co., Ltd., Beijing, China) was used for ultraviolet-visible absorption spectra. Fluorescence spectra were recorded by fluorescence spectrophotometer (Cary Eclipse, Varian, PaloAlto, CA, USA). ECL experiments were carried out by CHI660E electrochemical workstation (Shanghai Chenhua instrument Co., Ltd.) and RFL-1 bioluminescence detection/ultra-weak chemiluminescence detector (Xi'an Ruimai Analytical Instrument Co., Ltd.).

\section{Preparation of PALNWS}

The preparation steps of PALNWs were as follows: firstly, $2 \mathrm{~mL}\left(0.05 \mathrm{~mol} \mathrm{~L}^{-1}\right)$ aniline sulfate solution, $2 \mathrm{~mL}\left(2 \mathrm{mg} \mathrm{mL}^{-1}\right)$ luminol alkaline solution and $1 \mathrm{~mL}(1 \mathrm{~mol}$ $\mathrm{L}^{-1}$ ) sulfuric acid solution were added to a clean conical bottle and sonicated for 40 
minutes. After the solution was cooled to room temperature, $2 \mathrm{~mL}\left(0.05 \mathrm{~mol} \mathrm{~L}^{-1}\right)$ ammonium persulfate solution was slowly added to the above solution as an initiator and stirred at room temperature for 7 hours to obtain a dark green mixed solution. The mixed solution was centrifuged with $12000 \mathrm{r} / \mathrm{min}$ for 10 minutes, and the unreacted reagents were removed. The obtained product was washed 3 times at the same speed, 10 minutes each time, and the residues wash to neutral. Finally, the obtained PALNWs were dispersed in $2 \mathrm{~mL}$ ultrapure water and kept away from light at $4{ }^{\circ} \mathrm{C}$ in the refrigerator.

\section{Preparation of the ECL DNA biosensor}

The preparation process of ECL DNA biosensor based on PALNWs were as follows: First, $30 \mu \mathrm{L}$ PALNWs were dropped on the surface of a ITO electrode and dried at room temperature. $10 \mu \mathrm{L} 0.25 \%$ GA solution was added on the surface of the PALNWs ITO electrode as a cross-linking agent, and then kept it for 2 hours at $4{ }^{\circ} \mathrm{C}$; Then the modified electrode was washed with $0.01 \mathrm{~mol} \mathrm{~L}^{-1}$ phosphate buffer solution $(\mathrm{PBS})(\mathrm{pH}=7.4)$ to remove excess unbound reagents; Then $20 \mu \mathrm{L}$ of $1 \mu \mathrm{mol} \mathrm{L}{ }^{-1}$ amination cDNA was dropped on the surface of the modified electrode and incubated at $4{ }^{\circ} \mathrm{C}$ for 12 hours. During this process, cDNA was covalently linked onto the surface of the PALNWs via glutaraldehyde crosslinkage. After that, the modified electrode was rinsed with $0.01 \mathrm{~mol} \mathrm{~L}^{-1} \mathrm{PBS}$ to remove the unbound cDNA; Then, the cDNA/PALNWs/ITO electrode was obtained.

\section{ECL detection for target DNA}

The hybridization process was performed by dropping $20 \mu \mathrm{L}$ aliquots of hybridization buffer solution containing various concentrations of tDNA and Fc-DNA on cDNA/PALNWs/ITO electrode and incubated at $37{ }^{\circ} \mathrm{C}$ for 1 hour, and followed by a thorough washing with the same buffer to remove any unbound tDNA and Fc-DNA. The modified electrodes were taken in borax buffer solution $(\mathrm{pH} 9.18)$ under a pulse potential for a typical ECL test. The ECL intensity difference $\Delta I$ in the absence and presence of tDNA under pulse potential was used for quantification.

\section{Results and Discussion}

\section{Characterization of PALNWS}

Luminol has been modified on electrode surface by various methods to fabricate reagentless-based ECL sensor and to broaden the application fields of ECL-based sensor. ${ }^{28,29}$ In this work, PALNWs were synthesized by a simple one-step synthesis method, and the morphology of the PALNWs were studied by transmission electron microscope (TEM). As shown in Figure 1A and B, it can be seen that the nanowires are linear structure, and the average diameter of the nanowires is about $20 \sim 30 \mathrm{~nm}$.

Figure 2 showed the UV-vis absorption spectra of PANI, luminol and PALNWs dispersed in aqueous solution respectively. Polyaniline (curve a) showed a weak absorption peak at $350 \mathrm{~nm}$, which is due to the $\pi \rightarrow \pi^{*}$ electron transition of benzene 
ring in PANI chain, a weak absorption peak at $450 \mathrm{~nm}$ and a tail peak at $700 \sim 800 \mathrm{~nm}$ which corresponds to the $\pi \rightarrow \pi^{*}$ transition and exciton-like transition from the benzenoid rings to the quinoid rings, respectively. ${ }^{30}$ These showed that PANI was successfully synthesized in the presence of sulfuric acid and ammonium persulfate. The spectrum of luminol (curve b) contained characteristic peaks at $220 \mathrm{~nm}, 300 \mathrm{~nm}$ and $350 \mathrm{~nm}$, respectively, this was consistent with the report in the literature. ${ }^{31}$ The spectrum of PALNWs displayed absorption peaks of polyaniline and luminol, which indicated the existence of the two substances. The experimental results showed that luminol and aniline were successfully polymerized together to form nanowires.

The inset of Figure 2 showed the fluorescence spectra of polyaniline, luminol and PALNWs that were dispersed in $0.1 \mathrm{~mol} \mathrm{~L}^{-1}$ potassium carbonate solution. Fluorescence measurement was carried out at $350 \mathrm{~nm}$ as the excitation wavelength. It can be seen that polyaniline (Inset of Figure 2, curve a) has no obvious fluorescence emission. The fluorescence spectra of luminol displayed maximum fluorescence emission at $410 \mathrm{~nm}$. Furthermore, the PALNWs showed obvious fluorescence emission with a maximum fluorescence emission $420 \mathrm{~nm}$. According to the result, it can be inferred that the fluorescence of the PALNWs comes from the polymerized luminol in the nanowires, which proved the existence of luminol in the nanowires. It is also important to note that the maximum emission wavelength has a significant red shift compared with luminol. The reason for the red shift may be that the fluorescence of luminol in nanowires was affected by its concentration and matrix property.

\section{ECL behavior of PALNWS}

The ECL behavior of PALNWs modified ITO electrode was studied under cyclic voltammetry in borax buffer solution ( $\mathrm{pH}$ 9.18). The curves of ECL intensity versus applied potential (Figure 3, curve a) and the cyclic voltammogram (Figure 3, curve b) were recorded simultaneously. When the potential was swept from 0 to $1.0 \mathrm{~V}$ at the scanning rate of $0.1 \mathrm{~V} \mathrm{~s}^{-1}$, ECL signal started at about $0.6 \mathrm{~V}$, and with the increase of potential, the ECL intensity increased until the potential reached to $1.0 \mathrm{~V}$ and began to sweep back. The cyclic voltammogram showed that the PALNWs did not show obvious electrochemical redox peak due to the poor electrochemical activity of polyaniline and luminol in alkaline solution.

The ECL behavior of PALNWs modified ITO electrode in saturated borax buffer was also studied under pulse potential. As shown in the inset of Figure 3, when pulse potential was applied to the PALNWs modified ITO electrode, the ECL signals of the PALNWs for ten times are stable and strong. In addition, the ECL signals of the nanowires were measured for 100 periods, and the relative standard deviation (RSD) was $1.2 \%$. The results imply that luminol molecules were effectively polymerized with aniline to form nanowires and retained good luminescence properties, which may be used as a platform for the construction of sensor. The ECL emission originated from the reaction between the electrochemical product of luminol and dissolved oxygen. ${ }^{32}$

\section{Construction and characterization of ECL biosensor based on PALNWS}

In the experiment, it was found that the PALNWs can form a stable nanowires film 
without the assistance of other reagents or materials. Therefore, it is favorable for the construction of ECL-based biosensor combining the stable ECL signal and abundant amino groups of the PALNWs. On the other hand, inspired by the advantages of the sandwich type biosensors, a sensitive ECL DNA biosensor was designed using the PALNWs as sensing platform. Scheme 1 shows the two fabrication and sensing strategies for the construction of biosensors. In the process, PALNWs were used as ECL sensing platform and to immobilize cDNA through glutaraldehyde cross-linking. PALNWs were first immobilized on ITO electrode surface, and then, cDNA was immobilized on the electrode surface by glutaraldehyde crosslinking. In the first protocol, tDNA and Fc-DNA were successively modified on the electrode surface through hybridization, leading to a traditional sandwich structure. In the second protocol, the cDNA/GA/PALNWs/ITO electrode was immersed into the solution containing tDNA and Fc-DNA; In presence of tDNA, its $3^{\prime}$ terminus hybridized with the cDNA and the 5' terminus hybridized with the Fc-DNA. When the concentration of tDNA increased, Fc-DNA continuous hybridized with tDNA, resulting the supersandwich structure of long DNA concatamer on ITO electrode surface. ECL was employed to monitor the DNA hybridization event by measuring the ECL signal intensity of PALNWs. The Ferrocene on the Fc-DNA can effectively catalyze the ECL reaction of luminol, resulting enhanced ECL signal. The concentration of the tDNA was quantified based on the relationship between the concentration of tDNA and the enhanced ECL intensity.

Electrochemical impedance spectroscopy (EIS) was used to study the assembly process of the ECL biosensor in PBS $\left(0.1 \mathrm{~mol} \mathrm{~L}^{-1}, \mathrm{pH}=7.0\right)$ solution containing $5 \times 10^{-3}$ mol L $\mathrm{L}^{-1}\left[\mathrm{Fe}(\mathrm{CN})_{6}\right]^{3-/ 4-}$. Figure $4 \mathrm{~A}$ showed the EIS curves of the ECL biosensor constructed by the two strategies at different assembly stages. It can be seen the $R_{e t}$ value of PALNWs modified electrode (curve b) decreased compared to the bare ITO electrode (curve a), which may be due to the good conductivity and large specific surface area of the nanowires. Then, the electron transfer ability decreased and the resistance increased when GA was modified on the PALNWs electrode surface (curve c). Later, the addition of cDNA (curve d), tDNA and Fc-DNA on the electrode surface also led to the increase of charge transfer resistance in turn, which may be due to the electrostatic repulsion of the negatively charged DNA that hindered the electron transfer of $\left[\mathrm{Fe}(\mathrm{CN})_{6}\right]^{3-/ 4-}$ at the electrode interface. Compared with the traditional sandwich strategy (curve e), the impedance of the supersandwich strategy (curve f) increased more obviously. The reason is mainly that the long-chain DNA with larger negative charge had a greater electrostatic effect on $\left[\mathrm{Fe}(\mathrm{CN})_{6}\right]^{4-}$ in solution, and the blocking effect was more obvious. The results demonstrated that the electrode modified with sensing interface were fabricated as expected.

The ECL behaviors of the electrode at different modification stages were studied under pulse potential. Figure 4B showed that the bare ITO electrode showed no ECL signal (curve a) and PALNWs/ITO electrode displayed strong ECL signals (curve b); When GA was immobilized on PALNWs, ECL intensity decreased (curve c). Next, the immobilization of the cDNA on the electrode surface also led to the decrease of ECL (curve d). In the presence of Fc-DNA, there was no obvious change for the ECL of the 
cDNA/GA/PALNWs/ITO electrode, indicating that hybridization reaction did not occur between cDNA and Fc-DNA. In the presence of target DNA and Fc-DNA, hybridization occurred between capture DNA, target DNA and Fc-DNA to form FcDNA/tDNA/cDNA/GA/PALNWs/ITO electrode according the proposed two strategies, the ECL intensity both increased obviously (curve e and f) due to the catalysis of the Ferrocene on the ECL reaction of luminol. Furthermore, by comparison, the ECL intensity of $\mathrm{Fc}-\mathrm{DNA} / \mathrm{tDNA} / \mathrm{cDNA} / \mathrm{GA} / \mathrm{PALNW} / \mathrm{ITO}$ electrode obtained by the traditional sandwich strategy (the curve e) did not increase as much as that of the supersandwich strategy (the curve f). This was mainly because each tDNA chain can only achieve a single binding pair in the traditional sandwich strategy, while in the supersandwich strategy, the tDNA chain can achieve a pairing reaction between two different regions, which can be combined with more Fc-DNA, and Ferrocene can catalyze ECL reaction of luminol. As a result, a higher ECL response signal was generated. In addition, compared with the curve $d$, in the absence of Fc-DNA, the ECL intensity of tDNA/cDNA/GA/PALNWs/ITO (curve g) was obviously weakened, which was mainly due to that double stranded DNA formed by the hybridization of cDNA and tDNA hindered the electron transfer of electrode and the ECL reaction of luminol. The mechanism of the ECL is speculated as follows: under electrolysis, Fc can be oxidized to $\mathrm{Fc}^{+}$, and luminol can be oxidized by $\mathrm{Fc}^{+}$to a high energy state. Therefore, luminol is more easily electro-oxidized to luminol radical anion (azasemiquinone); the luminol radical anion next reacted with the dissolved oxygen to produce excited 3aminophthalate anion, which emitted ECL when the excited 3-aminophthalate anion returned to the ground state. ${ }^{32,33}$

The immobilization process of ECL DNA biosensor was also studied by cyclic voltammetry in $0.01 \mathrm{~mol} \mathrm{~L}^{-1} \mathrm{PBS}(\mathrm{pH}=7.40)$ (cyclic voltammograms of different modified electrodes in Supporting Information S1, Figure S1), which approved the formation of the supersandwich structure. ${ }^{34}$

\section{Analytical performance of the ECL DNA biosensor}

Under the selected experimental conditions (Supporting Information, S2 and Figure S2), the ECL DNA biosensor constructed in this experiment was used to determine different concentrations of tDNA to evaluate the analytical performance. The analytical performances of the cDNA/GA/PALNWs/ITO electrode for tDNA with the two proposed strategies were both studied.

Figure 5A is the ECL response to the tDNA with the supersandwich strategy. The quantitative behavior of the fabricated ECL DNA biosensor was assessed by measuring the dependence of $\Delta I$ on the concentration of tDNA. The ECL intensity increased with an increase of the concentration of tDNA. The calibration curve for the determination of tDNA is shown in the inset of Figure $5 \mathrm{~A}$. $\Delta I$ was linear with the logarithm of concentration of tDNA over the range of $1.0 \times 10^{-16} \sim 1.0 \times 10^{-8} \mathrm{~mol} \mathrm{~L}^{-1}$. The linear regression equation was $\Delta I=19293.88+1227.13 \log C$ with a correlation coefficient of 0.9916. At the same time, the corresponding ECL signal of target DNA was measured in the range of low concentration, the linear regression equation was $\Delta I=357.75+$ $5.79 \times 10^{17} \mathrm{C}$. The limit of detection (LOD) for the tDNA was $5.8 \times 10^{-17} \mathrm{~mol} \mathrm{~L}^{-1}$, which 
was calculated according to the formula $\mathrm{LOD}=3 \mathrm{~s}_{\mathrm{b}} / \mathrm{S}(\mathrm{S} / \mathrm{N}=3)$, where $\mathrm{s}_{\mathrm{b}}$ is the standard deviation of the blank signal $\left(\mathrm{n}_{\mathrm{b}}=11\right)$ and $\mathrm{S}$ is the analytical sensitivity, which can be estimated as the slope of the calibration plot at lower concentrations (Figure 5B and the inset of Figure 5B). In addition, the analytical performance of the proposed ECL DNA biosensor with the traditional sandwich strategy was also investigated (Supporting Information, S3 and Figure S3). The results showed that this ECL biosensor with the supersandwich strategy has higher sensitivity, a wider linear range, and a lower detection limit than that of the ECL biosensor with the traditional sandwich strategy.

In order to evaluate the specificity of the prepared ECL DNA biosensor, the specificity of the developed ECL biosensor was investigated by introducing single base mismatch sequence or non-complementary sequence into the solution instead of the target sequence (tDNA). The experimental results were shown in Figure 6. It can be seen that the ECL increased significantly in the presence of tDNA, and there was no significant difference in ECL intensity with single base mismatch sequence or noncomplementary sequence. Consequently, the ECL biosensor had good selectivity and could achieve specific determination for tDNA.

Furthermore, a comparison between the proposed ECL biosensor and other previously reported biosensor for tDNA was made (Table 1), and the result showed that this ECL biosensor has higher sensitivity, wider linear and lower detection limit than the other developed biosensors. Furthermore, the proposed ECL biosensor can be prepared easily due to the excellent film-forming of PALNWs on ITO electrode and simple DNA hybridizing strategy, which made it possible for mass production.

\section{Sample analysis}

In order to evaluate the applicability of the proposed method in real sample matrix, the standard addition method was used to add the standard solution into human urine samples. The biosensor prepared in this experiment was used to analyze the solution of the spiked sample. Human urine was diluted 10-fold with $\mathrm{pH}=7.4$ phosphate buffer solution, and then different concentrations of tDNA were added to the diluted urine. The recovery and relative standard deviation were determined by ECL and calculated. The results were shown in Table 2 . The recovery was in the range of $96.9 \% \sim 101.3 \%$, and the relative standard deviation was between 3.8\% 5.1\%. These results indicated that the ECL DNA biosensor constructed by the PALNWs had good reliability and had certain feasibility in the detection of tDNA in actual urine samples.

\section{Conclusions}

In this experiment, PALNWs were synthesized by a simple and rapid chemical oxidation method, which showed good film-forming ability and stable ECL property on electrode surface. On the basis of the prepared PALNWs, a sensitive signal-on ECL supersandwich DNA biosensor was developed combining the long DNA concatamer formation and the catalysis of Ferrocene on ECL reaction of luminol. Through the base complementary pairing reaction between the tDNA, the capture DNA and Fc-DNA, the 
quantitative detection were realized for the one fragment of E. coli DNA sequence (tDNA). The ECL DNA biosensor had the advantages of strong selectivity, simple preparation and sensitive response to the one fragment of E. coli DNA sequence. Furthermore, this strategy can also be used in the chemiluminescence determination of other biomarkers based on the good stability of PALNWs on solid support.

\section{Acknowledgements}

This research was financially supported by the National Natural Science Foundation of China (No. 21665025) and College Students Innovation and Entrepreneurship Training Project of Xinjiang Normal University (No. X2020107622019), which are gratefully acknowledged. 


\section{References}

1. A. Kowalczyk, Curr. Opin. Electroche., 2020, 23, 36.

2. E. O. Blair, D. K. Corrigan, Biosens. Bioelectron., 2019, 134, 57.

3. C. Park, H. Park, H. J. Lee, H. S. Lee, K. H. Park, C. H. Choi and S. Na, Microchim. Acta, 2018, 186, 34.

4. L. Lan, Y. Yao, J. Ping and Y. Ying, Sens. Actuator B-Chem., 2019, $290,565$.

5. T. K. Mandal, N. Parvin, K. Mishra, S. Mohandoss and Y. R. Lee, Microchim. Acta, 2019, 186, 833.

6. M. Dadmehr, M. A. Karimi and B. Korouzhdehi, Spectroc. Acta Pt. A-Molec. Biomolec. Spectr., 2020, 228, 117731.

7. X. Yang, Q. Liu, D. Wen, M. Gao, D. Zhang, Q. Jin, J. Kong and J. Zhang, Anal. Chim. Acta, 2019, 1088, 144.

8. M. Zhang, H. Hai, F. Y. Zhou, J. C. Zhong and J. P. Li, Chin. J. Anal. Chem., 2018, 46, 203.

9. G. Li, S. Qiu, Y. Zhang, M. Li and M. Guan, Anal. Lett., 2018, 52, 1112.

10. X. P. Liu, J. S. Chen, C. J. Mao, H. L. Niu, J. M. Song and B. K. Jin, Biosens. Bioelectron., 2018, 116, 23.

11. Y. Gao, S. Xu, T. He, J. Li, L. Liu, Y. Zhang, S. Ge, M. Yan, H. Liu and J. Yu, Sens. Actuator B-Chem., 2020, 324, 128693.

12. X. J Wang, M. Yang, Q. Y Liu, S. Y. Yang, X. T Geng, Y. X Yang, H. B, Fa, Y. Z Wang and C. J. Hou, Anal. Sci., 2019, 355, 441.

13. F. Zhao, Y. Bai, L. Cao, G. Han and Z. Chen, J. Electroanal. Chem., 2020, 867, 114184.

14. X. Lin, X. Lian, B. Luo and X. C. Huang, Inorg. Chem. Commun., 2020, 119, 108095.

15. Z. Shi, G. Li and Y. Hu, Chin. Chem. Lett., 2019, 30, 1600.

16. Y. Chen, L. Sun, X. Qiao, Y. Zhang, Y. Li and F. Ma, Anal. Chim. Acta, 2020, 1103, 67.

17. H. Wu, Y. Su, J. Jiang, Y. Liang and C. Zhang, J. Lumines., 2020, 226, 117485.

18. X. Ou, C. Fang, Y. Fan, H. Chen, S. Chen and S. Wei, Sens. Actuator B-Chem., 2016, $228,625$.

19. K. L. Lin, T. Yang, F. F. Zhang, G. Lei, H. Y. Zou, Y. F. Li and C. Z. Huang, J. Mat. Chem. B, 2017, 5, 7335 .

20. S. Hanif, S. Han, P. John, W. Gao, S. A. Kitte and G. Xu, Electrochim. Acta, 2016, 196, 245.

21. A. Sassolas, L. J. Blum and B. D. Leca-Bouvier, Sens. Actuator B-Chem., 2009, $139,214$.

22. G. F. Zhang, H. Y. Chen, Anal. Chim. Acta, 2000, 419, 25.

23. Y. Wang, S. Hamid, X. Zhang, N. Akhtar, X. Zhang and T. He, New J. Chem., 2017, $41,1591$.

24. G. Li, J. Lian, X. Zheng and J. Cao, Biosens. Bioelectron., 2010, 26, 643.

25. C. Wang, L. Chen, P. Wang, M. Li and D. Liu, Biosens. Bioelectron., 2019, 133, 154. 
26. L. Wei, Y. Zhang, N. Eziz, Y. Yang, G. Li and M. Guan, Anal. Bioanal. Chem., 2019, 411, 5175.

27. N. Shoaie, M. Daneshpour, M. Azimzadeh, S. Mahshid, S. M. Khoshfetrat, F. Jahanpeyma, A. Gholaminejad, K. Omidfar and M. Foruzandeh, Microchim. Acta, 2019, 186, 465.

28. Y. Pu, M. Zhou, P. Wang, Q. Wu, T. Liu and M. Zhang, J. Electroanal. Chem., 2020, 873, 114374.

29. C. Fang, H. Li, J. Yan, H. Guo and T. Yifeng, ChemElectroChem, 2017, 4, 1587.

30. A. Saikia and N. Karak, Mater. Today Commun., 2018, 14, 82.

31. F. Li, W. Ma, J. Liu, X. Wu, Y. Wang and J. He, Anal. Bioanal. Chem., 2017, 410, 543.

32. K. E. Haapakka and J. J. Kankare, Anal Chim Acta, 1982, 138: 263.

33. F. Li, Y. Yu, Q. Li, M. Zhou and H. Cui, Anal. Chem. 2014, 86, 3, 1608.

34. F. Xia, R. J. White, X. Zuo, A. Patterson, Y. Xiao, D. Kang, X. Gong, K. W. Plaxco, and A. J. Heeger, J. Am. Chem. Soc., 2010, 132, 14346.

35. Y. Chen, J. Xu, J. Su, Y. Xiang, R. Yuan and Y. Chai, Anal. Chem., 2012, 84, 7750.

36. Q. Ma, Y.-L. Li, N. C. Gong, X. Jiang and S. Y. Huan, Chin. J. Anal. Chem., 2015, 43, 1676.

37. F. Gao, J. Lei and H. Ju, Anal. Chem., 2013, 85, 11788.

38. C. Song, X. Yang, K. Wang, Q. Wang, J. Huang, J. Liu, W. Liu and P. Liu, Anal. Chim. Acta, 2014, 827, 74.

39. R. Liu, C. Wang, Y. Xu, J. Hu, D. Deng and Y. Lv, Anal. Chem., 2017, 89, 13269.

40. J. Su, H. Zhang, B. Jiang, H. Zheng, Y. Chai, R. Yuan and Y. Xiang, Biosens. Bioelectron., 2011, 29, 184.

41. Q. Wang, J. Su, J. Xu, Y. Xiang, R. Yuan and Y. Chai, Sens. Actuator B-Chem., 2012, 163, 267. 
Table1 Comparison of the developed ECL biosensor for target DNA with other biosensor from the previous literatures.

\begin{tabular}{cccc}
\hline Detection Method & $\begin{array}{c}\text { Linear Rang/ } \\
(\mathrm{mol} / \mathrm{L})\end{array}$ & $\begin{array}{c}\text { Detection Limit/ } \\
(\mathrm{mol} / \mathrm{L})\end{array}$ & References \\
\hline ECL & $2.5 \times 10^{-14} \sim 1.0 \times 10^{-10}$ & $1.5 \times 10^{-14}$ & 35 \\
SERS & $5.0 \times 10^{-12} \sim 5.0 \times 10^{-9}$ & $5.0 \times 10^{-12}$ & 36 \\
SERS & $1.0 \times 10^{-10} \sim 1.0 \times 10^{-6}$ & $4.5 \times 10^{-11}$ & 37 \\
Fluorescence & $0 \sim 5.0 \times 10^{-10}$ & $4.0 \times 10^{-10}$ & 38 \\
ICPMS & $2.0 \times 10^{-11} \sim 1.0 \times 10^{-9}$ & $4.0 \times 10^{-12}$ & 39 \\
SWV & $5.0 \times 10^{-15} \sim 1.0 \times 10^{-13}$ & $5.0 \times 10^{-15}$ & 40 \\
SWV & $1.0 \times 10^{-12} \sim 1.0 \times 10^{-9}$ & $1.0 \times 10^{-12}$ & 41 \\
ECL(traditional & $1.0 \times 10^{-14} \sim 1.0 \times 10^{-8}$ & $3.1 \times 10^{-15}$ & This work \\
sandwich) & $1.0 \times 10^{-16} \sim 1.0 \times 10^{-8}$ & $5.8 \times 10^{-17}$ & This work \\
ECL(super sandwich) & & & \\
\hline
\end{tabular}

Table2 Determination results of target DNA in human urine samples

\begin{tabular}{ccccc} 
Samples & Added $/\left(\mathrm{mol} \mathrm{L}^{-1}\right)$ & Found $/\left(\mathrm{mol} \mathrm{L}^{-1}\right)$ & $\mathrm{RSD} / \%(\mathrm{n}=3)$ & Recovery $/ \%$ \\
\hline 1 & $1.0 \times 10^{-11}$ & $9.69 \times 10^{-12}$ & 3.8 & 96.9 \\
2 & $1.0 \times 10^{-12}$ & $1.01 \times 10^{-12}$ & 4.0 & 101.3 \\
3 & $1.0 \times 10^{-13}$ & $9.95 \times 10^{-14}$ & 5.1 & 99.5 \\
\hline
\end{tabular}




\section{Figure Captions}

Scheme. 1 Schematic description for the fabrication procedure of the proposed ECL DNA biosensor

Fig. 1 TEM image of the PALNWs (A and B).

Fig. 2 UV-vis spectrum of PANI(a), luminol (b), PALNWs (c). The inset shows fluorescence spectra of PANI(a), luminol (b), PALNWs (c).

Fig. 3 ECL curve versus potential (curve a) and cyclic voltammogram of PALNWs (curve b) in borax buffer solution (pH 9.18). The inset shows ECL signals of PALNWs under pulse potential for 10 times. Experimental parameters of pulse potential: initial potential, $0 \mathrm{~V}$; pulse potential, $0.9 \mathrm{~V}$; pulse time, $0.1 \mathrm{~s}$; pulse period, $30 \mathrm{~s}$.

Fig. 4 EIS (A) and ECL signals (B) of bare ITO electrode (a), PALNWs/ITO electrode (b), GA/PALNWs/ITO electrode (c), cDNA/GA/PALNWs/ITO electrode (d), traditional sandwich Fc-DNA/tDNA/cDNA/GA/PALNWs/ITO electrode (e), supersandwich Fc-DNA/tDNA/cDNA/GA/PALNWs/ITO electrode (f), tDNA/cDNA/GA/PALNWs/ITO electrode (g);

Fig. 5 (A) ECL signals at different tDNA concentrations with the supersandwich strategy. Inset: linear relationship between ECL response and logarithm of tDNA concentration with the supersandwich strategy; (B) ECL signals at lower concentration of tDNA; Inset: calibration curve of tDNA at lower concentrations.

Fig. 6 Histograms of the ECL responses of blank (a), single base mismatch sequence (b), non-complementary sequence (c), target sequence with traditional sandwich strategy (d) and target sequence with supersandwich strategy (e). 


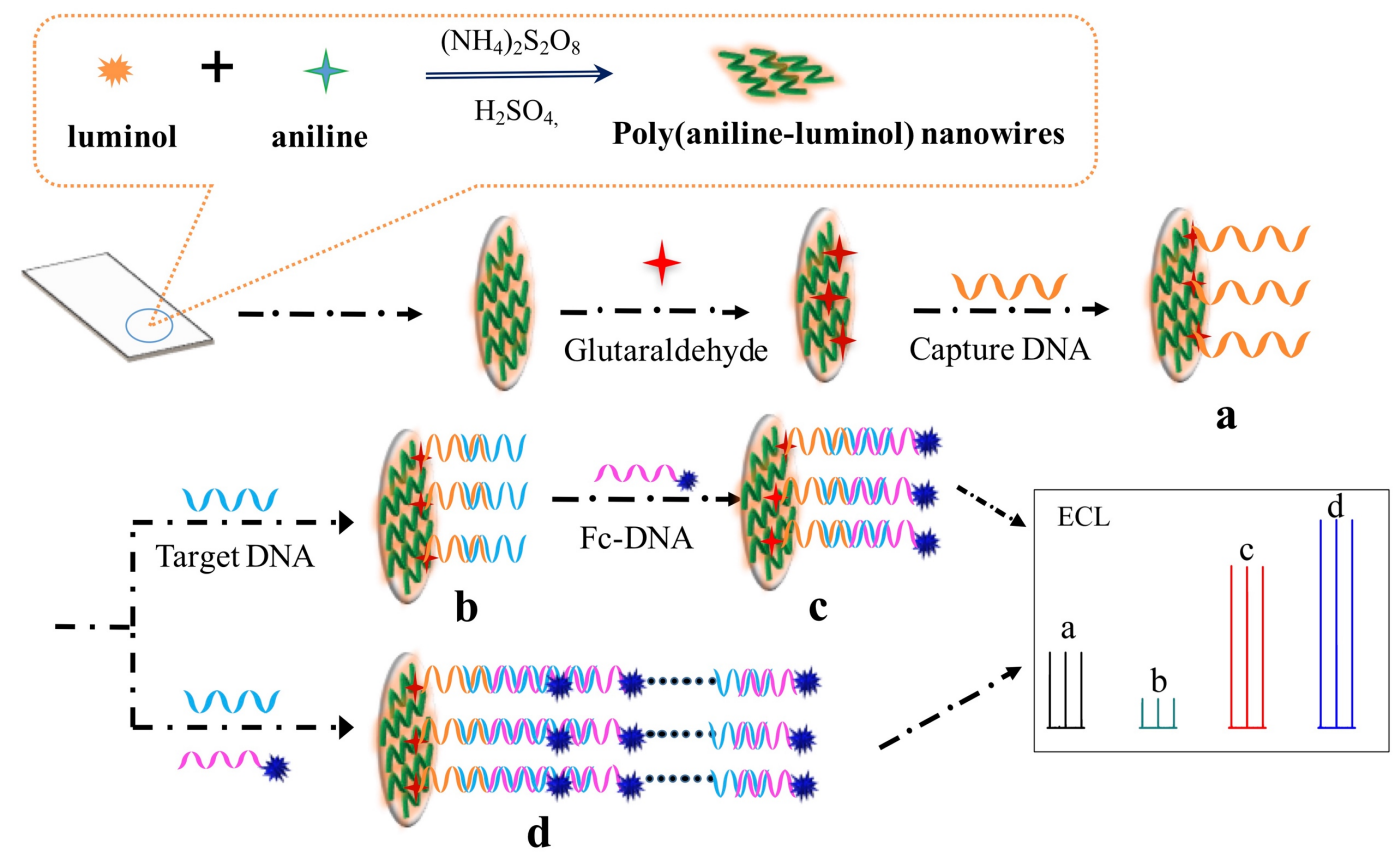

Scheme. 1 

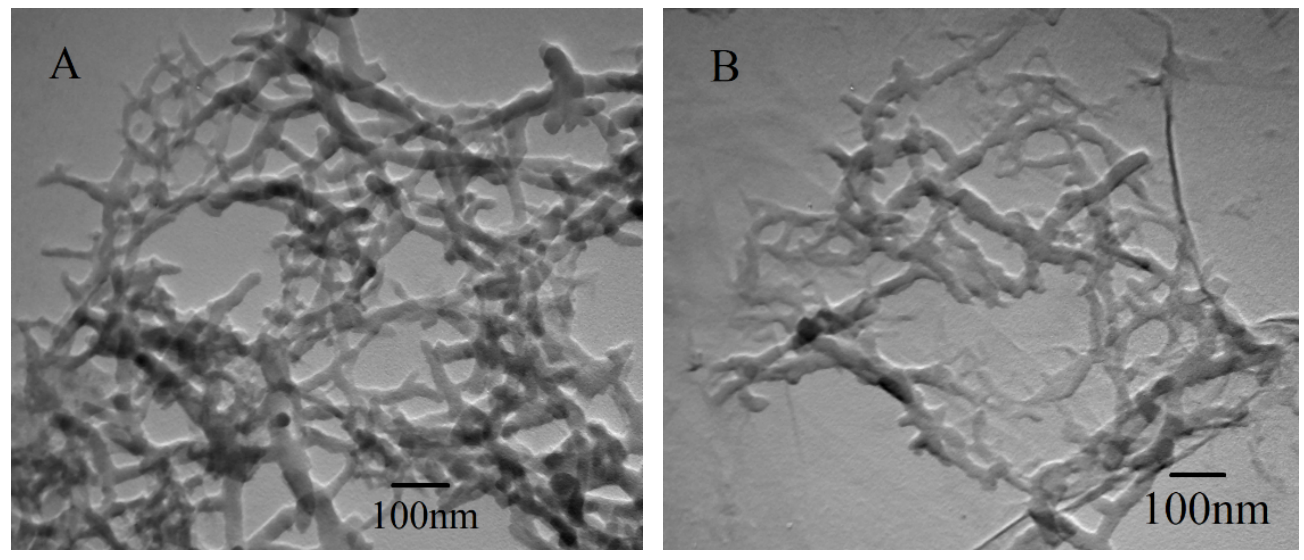

Fig. 1

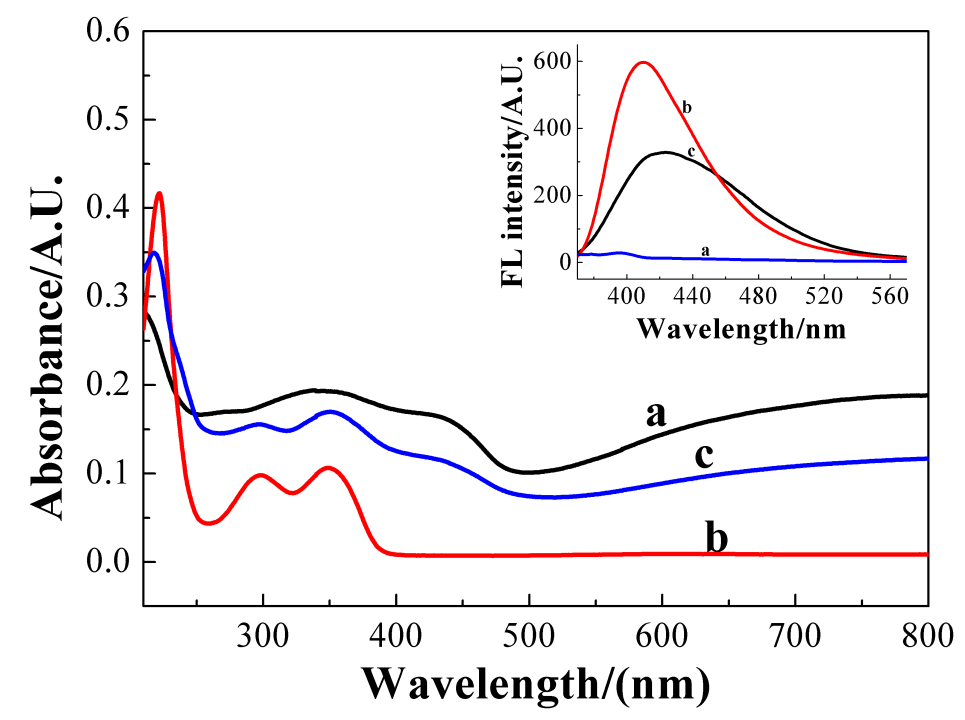

Fig. 2 


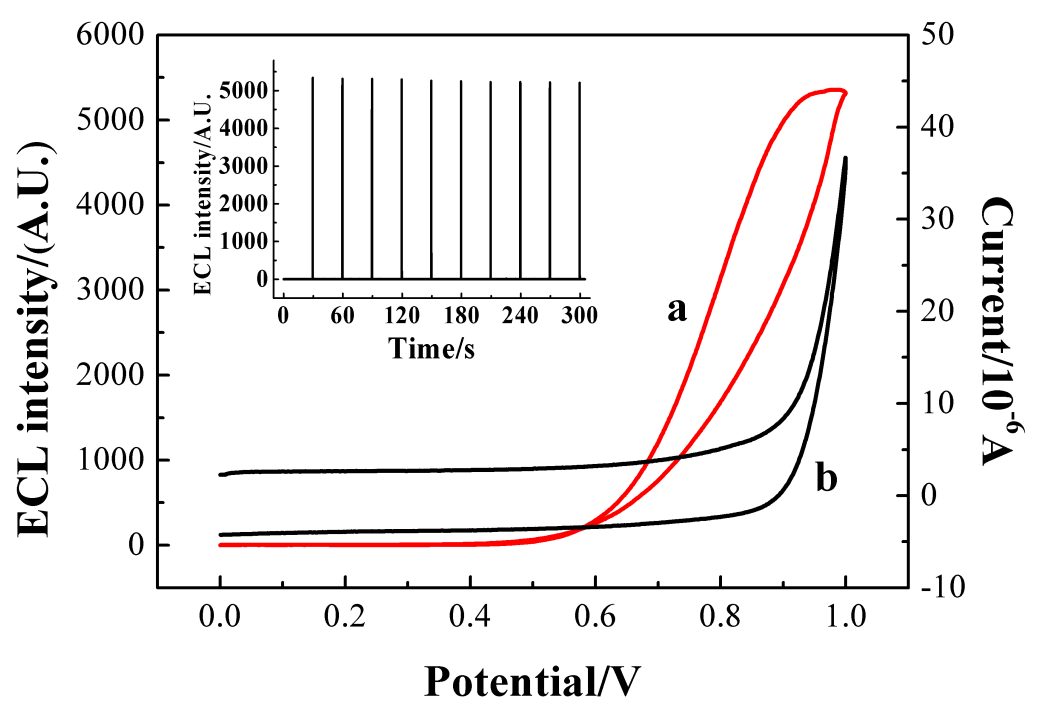

Fig. 3
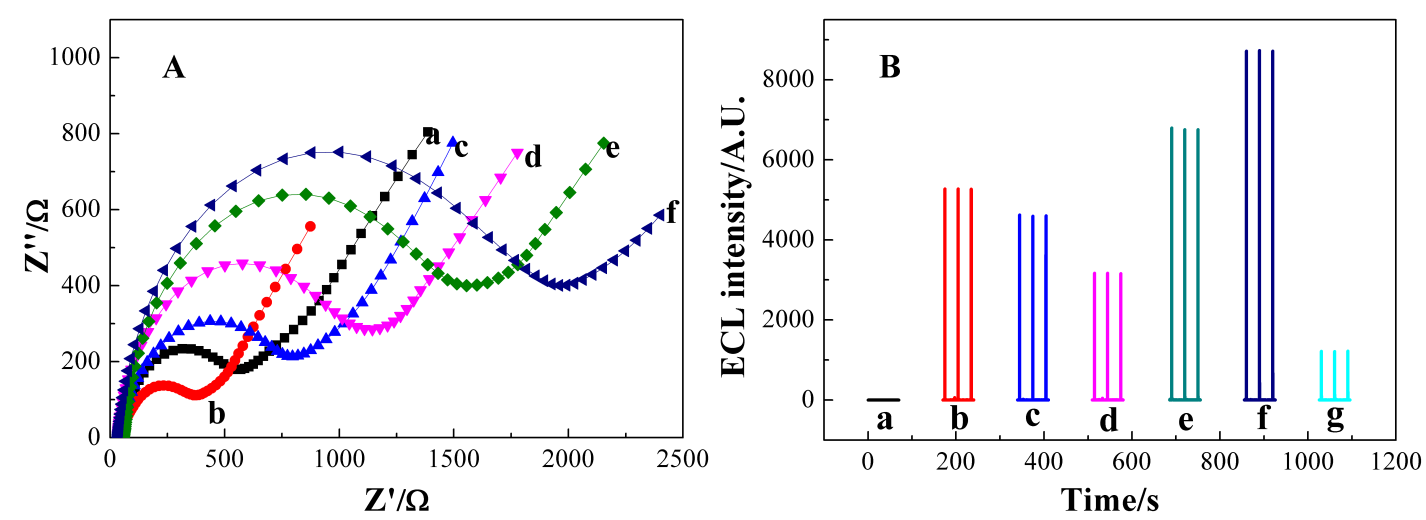

Fig. 4
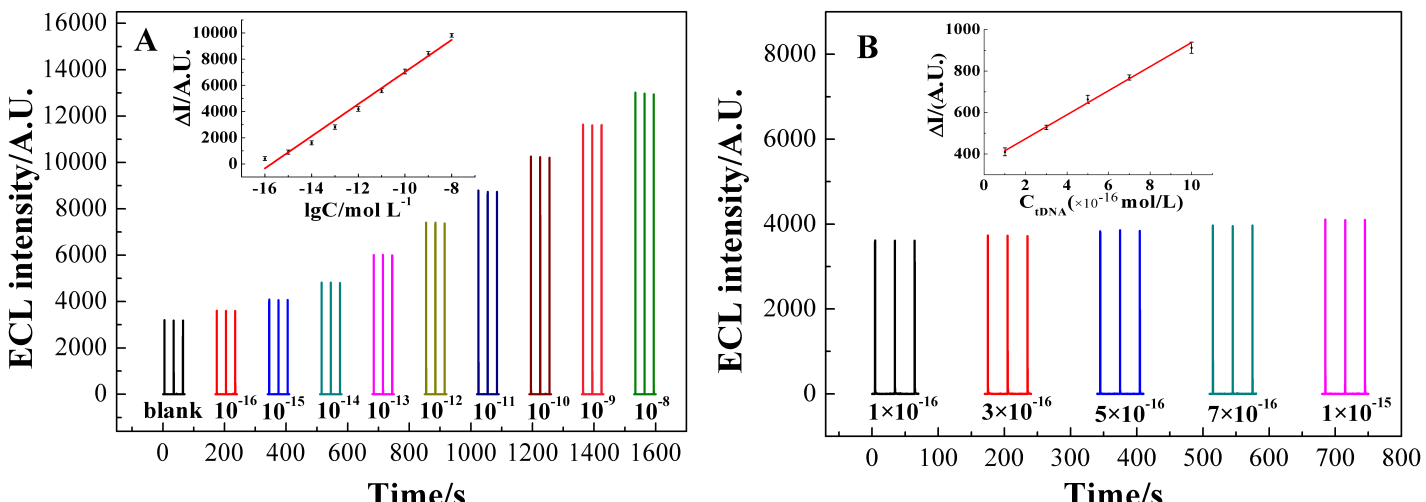

Fig. 5 


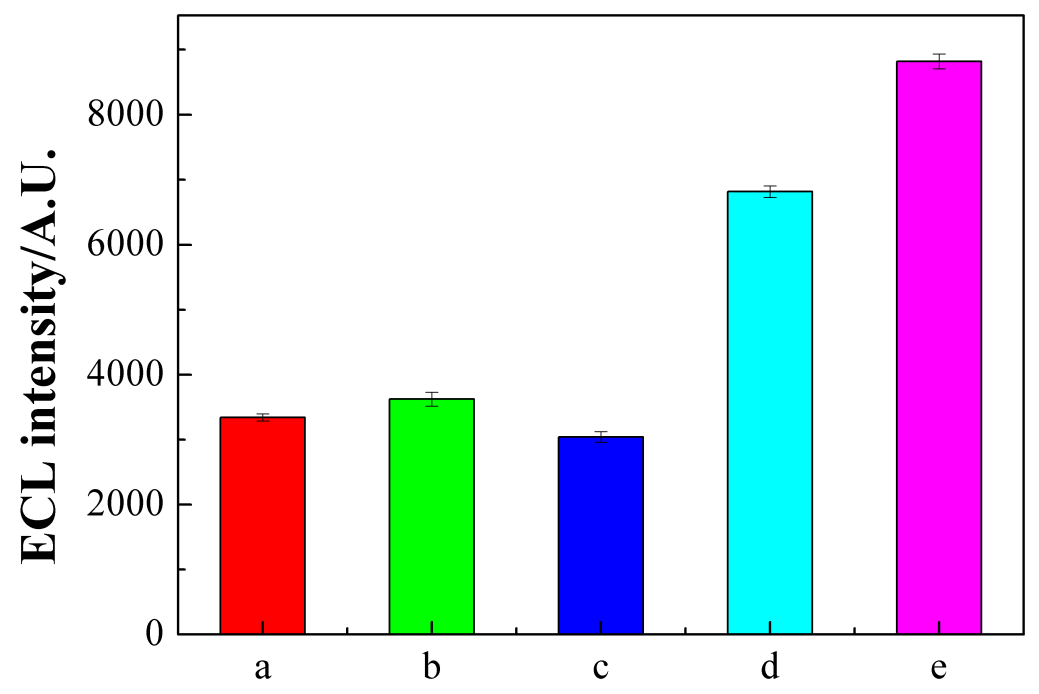

Fig. 6 


\section{Graphical Index}

The figure for the Graphical Index saved in the JPEG, PNG or TIFF format at 300 dpi should be pasted with the size adjusted to the frame below $(5 \mathrm{~cm}$ long and $8 \mathrm{~cm}$ wide).

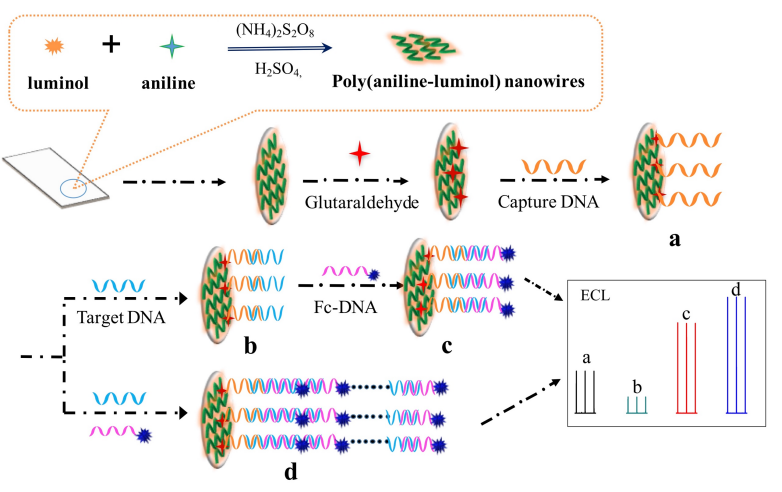

